\title{
FAMILIES OF PARTIAL REPRESENTING SETS
}

\author{
KEVIN P. BALANDA
}

(Received 17 December 1982)

Communicated by J. N. Crossley

\begin{abstract}
Assume GCH. Let $\kappa, \lambda, \mu, \Sigma$ be cardinals, with $\kappa$ infinite. Let $\mathcal{Q}$ be a family consisting of $\lambda$ pairwise almost disjoint subsets of $\Sigma$ each of size $\kappa$, whose union is $\Sigma$. In this note it is shown that for each $\mu$ with $1 \leqslant \mu \leqslant \min (\lambda, \Sigma)$, there is a "large" almost disjoint family $\mathcal{T}$ of $\mu$-sized subsets of $\Sigma$, each member of $\mathcal{T}$ having non-empty intersection with at least $\mu$ members of the family $\mathbb{Q}$.
\end{abstract}

1980 Mathematics subject classification (Amer. Math. Soc.): 04 A 20.

\section{Introduction}

If $\lambda$ and $\kappa$ are cardinals, a $(\lambda, \kappa)$ family is an indexed family $\left(S_{i} ; i \in I\right)$ of sets where $|I|=\lambda$ and $\left|S_{i}\right|=\kappa$ for each $i$ in $I$.

A family $\mathscr{X}$ of sets is said to be almost disjoint if $\left|X \cap X^{\prime}\right|<\min \left(|X|,\left|X^{\prime}\right|\right)$ for all pairs $X, X^{\prime}$ of elements of $\mathscr{X}$. The degree of disjunction, $\delta(\mathscr{X})$, of the family $\mathscr{X}$ is the least cardinal $\theta$ such that $\left|X \cap X^{\prime}\right|<\theta$ for all pairs $X, X^{\prime}$ of elements of $\mathfrak{X}$. A set $T$ is called a representing set of $\mathscr{X}$ if $T \subseteq \cup \mathcal{X}$ and $T \cap X \neq \varnothing$ for each $X$ in $\mathcal{X}$.

Suppose $\kappa$ is an infinite cardinal and $\mathcal{Q}$ is an almost disjoint family of $\kappa$-sized sets. In Balanda [1] it was shown (assuming $G C H$ ) that $\mathbb{Q}$ need not possess an almost disjoint pair of representing sets if $|\mathscr{Q}|>\kappa$. Almost disjoint families of representing sets are studied further in Balanda [2]. This paper is concerned with families of sets, each of which is a representing set of some fixed sized subfamily of $\mathcal{Q}$.

The Generalized Continuum Hypothesis (GCH) is assumed throughout the general discussion.

(C) 1985 Australian Mathematical Society $0263-6115 / 85 \$ A 2.00+0.00$ 
Suppose $\kappa$ is an infinite cardinal and $\mathscr{Q}$ is an almost disjoint $(\lambda, \kappa)$ decomposition of the cardinal $\Sigma$. If $1 \leqslant \mu \leqslant \min (\lambda, \Sigma)$ then a $\mu$-sized representing set of some $\mu$-sized subfamily of $\mathcal{Q}$ is called a $\mu$-partial representing set of $\mathcal{Q}$. We are interested in the 'maximum' cardinality of an almost disjoint family of $\mu$-partial representing sets of $\mathfrak{Q}$. The following definition is useful.

Definition. Suppose $\theta, \mu$ are cardinals with $1 \leqslant \theta \leqslant \mu \leqslant \min (\lambda, \Sigma)$. Let

$R S_{\theta}(\mu, \mathscr{Q})=\sup \{|\mathcal{T}| ; \mathcal{T}$ is a family of $\mu$-partial representing sets of $\mathscr{Q}$ and $\delta(\mathcal{T}) \leqslant \theta\} . R S_{\mu}(\mu, \mathbb{Q})$ is often written $R S(\mu, \mathbb{Q})$.

Our aim is to establish the following theorem.

TheOREM. (GCH). Suppose $\mu, \lambda, \kappa, \Sigma$ are cardinals with $\kappa$ infinite, $\kappa \leqslant \Sigma$ and $1 \leqslant \mu \leqslant \min (\lambda, \Sigma)$. Let $\mathbb{Q}$ be an almost disjoint $(\lambda, \kappa)$ decomposition of $\Sigma$.

(i) If $\theta<\mu$ or if $\mu^{\prime} \neq \Sigma^{\prime}$, then $R S_{\theta}(\mu, \mathbb{Q})=\Sigma$

(ii) If $\mu^{\prime}=\Sigma^{\prime}$ then $R S(\mu, Q)=\Sigma^{+}$.

Moreover, the supremum in the definition of $R S_{\theta}(\mu, \mathbb{Q})$ is a maximum and not a strict supremum.

This theorem is proved in Section 2 in a series of propositions. The cardinal $R S_{\theta}(\mu, \mathfrak{Q})$ is 'as large as possible' in the following sense. Suppose $1 \leqslant \theta \leqslant \mu \leqslant \Sigma$ and $\Sigma$ is infinite, and let

$$
S_{\theta}(\mu, \Sigma)=\sup \left\{|\mathscr{X}| ; \mathscr{X} \subseteq[\Sigma]^{\mu} \text { and } \delta(\mathscr{X}) \leqslant \theta\right\} .
$$

It follows from Baumgartner [3] that $R S_{\theta}(\mu, \mathcal{Q})=S_{\theta}(\mu, \Sigma)$ always, and hence that $R S_{\theta}(\mu, \mathbb{Q})$ is as large as possible.

Our set notation is standard. An ordinal is identified with the set of its predecessors and cardinals are identified with initial ordinals. We use $\alpha, \beta, \gamma, \delta, \ldots$ to denote ordinals and $\lambda, \kappa, \Sigma, \mu, \theta, \ldots$ to denote cardinals. The cardinal $\kappa$ will always be infinite. If $\lambda$ and $\kappa$ are cardinals, a $(\lambda, \kappa)$ family is an indexed family $\left(S_{i}, i \in I\right)$ of sets where $|I|=\lambda$ and $\left|S_{i}\right|=\kappa$ for each $i$ in $I$. The symbol $[S]^{\mu}$ denotes $\left\{S^{\prime} ; S^{\prime} \subseteq S\right.$ and $\left.\left|S^{\prime}\right|=\mu\right\}$. The cofinality $\lambda^{\prime}$ of a non-zero cardinal $\lambda$ is the least cardinal $\mu$ such that $\lambda$ can be expressed as the sum of $\mu$ cardinals all less than $\lambda$. We say $\lambda$ is regular if $\lambda^{\prime}=\lambda$; otherwise $\lambda$ is singular in which case $\lambda^{\prime}<\lambda$. A $\lambda$-sequence is a sequence $\left\langle\lambda_{\sigma} ; \sigma<\lambda^{\prime}\right\rangle$ of cardinals all less than $\lambda$ such that $\lambda=\Sigma\left(\lambda_{\sigma} ; \sigma<\lambda^{\prime}\right)$. If $\lambda$ is singular then strictly increasing $\lambda$-sequences exist. An $\eta$-transversal of a family $\mathcal{X}$ is a subset $T$ of $\cup \mathcal{X}$ such that $1 \leqslant|T \cap X|<\eta$ for each $X$ in $\mathscr{X}$. A 2-transversal is called a transversal. If $\mathscr{X}=\left(X_{i}: i \in I\right)$ and $I^{\prime} \subset I$, then $\mathfrak{X}\left[I^{\prime}\right]$ denotes $\left(X_{i} ; i \in I^{\prime}\right)$. The family $\mathfrak{X}=\left(X_{i} ; i \in I\right)$ is said to be a $\Delta(\mu)$ family if $|I|=\mu$ and there is a set $K$ such that $X_{i} \cap X_{j}=K$ for all pairs 
$\{i, j\}$ in $[I]^{2}$. Such a system is called a delta family. The symbol

$$
(\lambda, \kappa) \rightarrow \Delta(\mu) \text { means: Every }(\lambda, \kappa) \text { family contains a } \Delta(\mu) \text { subfamily. }
$$

Delta families were studied in Erdos and Rado [4] and we refer the reader to this paper for details when needed. We refer the reader to Williams [5] for any further set theoretical background.

ACKNOWLEDGEMENT. The author would like to thank Dr. Neil H. Williams for the invaluable help and encouragement he gave while this work was being carried out.

\section{Proof of Theorem}

Throughout this section $\lambda, \kappa, \Sigma, \mu$ and $\theta$ denote non-zero cardinals such that $\kappa$ is infinite, $\kappa \leqslant \Sigma$ and $\theta \leqslant \mu \leqslant \min (\lambda, \Sigma)$. Note that although it follows that $\Sigma$ is infinite; neither $\lambda, \mu$ nor $\theta$ need be.

The first two results are concerned with the cardinalities of maximal families of $\mu$-partial representing sets. Note that every almost disjoint $\left(<\kappa^{\prime}, \kappa\right)$ family possesses a transversal.

LeMMA 1. (GCH). Suppose $\kappa<\Sigma$ and $\mu<\kappa^{\prime}$. Let $Q$ be an almost disjoint $(\lambda, \kappa)$ decomposition of $\Sigma$ and suppose $\mathcal{T}$ is a family of $\mu$-partial representing sets of $\mathbb{Q}$ such that $\delta(\mathscr{T}) \leqslant \theta$ and $|\mathscr{T}|<\Sigma$. Then $\mathscr{T}$ is not maximal with respect to $\delta(\mathscr{T}) \leqslant \theta$.

Proof. Write $\mathbb{Q}=\left(A_{\alpha} ; \alpha<\lambda\right)$. Since $\cup \mathbb{Q}=\Sigma$ and $\Sigma>\kappa$, it follows that $\lambda \geqslant \Sigma$. The conditions on the cardinals imply that $\mu<\Sigma$. Hence $|\cup \mathscr{T}|<\Sigma$ and $|\Sigma-\cup \mathscr{T}|=\Sigma$.

To show that $\mathscr{T}$ is not maximal we construct a $\mu$-sized subset $X$ of $\lambda$ and a $\mu$-sized transversal $T$ of $\mathscr{Q}[X]$ such that $T \cap \cup \mathcal{T}=\varnothing$. The construction of $X$ and $T$ depends on whether $\Sigma$ is regular or not.

Case 1. $\Sigma$ regular. Let $M=\left\{\alpha<\lambda ; A_{\alpha}-\cup \mathscr{T} \neq \varnothing\right\}$. Since $\Sigma-\cup \mathscr{T} \subseteq$ $\cup\left\{A_{\alpha} ; \alpha \in M\right\}$ it follows that $|M| \geqslant \Sigma$. We may assume, without loss of generality, that if $\{\alpha, \beta\} \in[M]^{2}$ then $A_{\alpha}-\cup \mathscr{T} \neq A_{\beta}-\cup \mathcal{T}$. For each $\alpha$ in $M$ we have $\left|A_{\alpha}-\cup \mathscr{T}\right| \leqslant \kappa$ and we partition the ordinals $\alpha$ in $M$ according to $\left|A_{\alpha}-\cup \mathscr{T}\right|$. Since $\Sigma$ is regular there is a set $M^{\prime}$ in $[M]^{\Sigma}$ and a cardinal $\rho$ with $1 \leqslant \rho \leqslant \kappa$ such that $\left|A_{\alpha}-\cup \mathcal{T}\right|=\rho$ for all $\alpha$ in $M^{\prime}$. If $\rho=\kappa$ choose $X$ from $\left[M^{\prime}\right]^{\mu}$. Then $\left(A_{\alpha}-\cup \mathcal{T} ; \alpha \in X\right)$ is an almost disjoint $(\mu, \kappa)$ family and choose $T$ to be a $\mu$-sized transversal of this family. The set $T$ is a $\mu$-sized transversal of $\mathscr{Q}[X]$ and $T \cap \cup \mathcal{T}=\varnothing$. If $\rho<\kappa$ then $\rho^{+}<\Sigma$ and $(\Sigma, \rho) \rightarrow \Delta(\mu)$, noting that $\mu<\Sigma$. (See 
Erdös and Rado [4].) Thus there is a set $X$ in $\left[M^{\prime}\right]^{\mu}$ such that ( $A_{\alpha}-\cup \mathscr{J} ; \alpha \in X$ ) is a $\Delta(\mu)$ system. Let $T$ be a $\mu$-sized transversal of this family. (This is possible because ( $\left.A_{\alpha}-\cup \mathscr{T} ; \alpha \in X\right)$ is a $\Delta(\mu)$ family of pairwise distinct sets.) Then $T$ is a $\mu$-sized transversal of $\mathscr{Q}[X]$ and $T \cap \cup \mathcal{T}=\varnothing$.

Case 2. $\Sigma$ singular. In this case let $L=\left\{\alpha<\lambda ;\left|A_{\alpha}-\cup \mathscr{J}\right|<\kappa\right\}$. Then $\left(A_{\alpha} \cap \cup \mathcal{T} ; \alpha \in L\right)$ is an almost disjoint $(|L|, \kappa)$ family of subsets of $\cup \mathscr{T}$ and $|L| \leqslant|\cup \mathscr{T}|^{+}<\Sigma$ since $|\cup \mathscr{T}|<\Sigma$ and $\Sigma$ is a limit cardinal. Hence $|\lambda-L|=\lambda$, and we choose $X$ from $[\lambda-L]^{\mu}$ and let $T$ be a $\mu$-sized transversal of the almost disjoint $(\mu, \kappa)$ family $\left(A_{\alpha}-\cup \mathcal{T}_{;} ; \alpha \in X\right)$. Then $T$ is a $\mu$-sized transversal of $\mathbb{Q}[X]$ and $T \cap \cup \mathcal{T}=\varnothing$.

This completes the proof of Lemma 1 .

Lemma 2. (GCH). Suppose $\kappa<\Sigma, \mu<\kappa^{\prime}, \mu$ is infinite and $\mu^{\prime}=\Sigma^{\prime}$. Let $\mathbb{Q}$ be an almost disjoint $(\lambda, \kappa)$ decomposition of $\sum$ and suppose $\mathscr{T}$ is an almost disjoint family

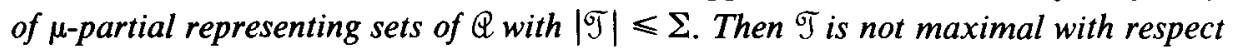
to almost disjointness.

Proof. Write $\mathcal{Q}=\left(A_{\alpha} ; \alpha<\lambda\right)$ and let $\mathscr{T}=\left(T_{\beta} ; \beta<\Sigma\right)$ where repetitions occur if $|\mathscr{T}|<\Sigma$. Note that the conditions on the cardinals imply that $\Sigma \leqslant \lambda, \mu<\Sigma$ and $\Sigma$ is singular. Let $\left\langle\mu_{\sigma} ; \sigma<\mu^{\prime}\right\rangle$ be a $\mu$-sequence and let $\left\langle\Sigma_{\delta} ; \delta<\mu^{\prime}\right\rangle$ be a strictly increasing $\Sigma$-sequence.

We construct sets $X$ from $[\lambda]^{\mu}$ and $T$ from $[\Sigma]^{\mu}$ such that $T$ is a transversal of $\mathscr{A}[X]$ and $\left|T \cap T_{\beta}\right|<\mu$ for each $\beta$ less than $\Sigma$. This establishes that $\mathcal{J}$ is not maximal.

Inductively, define a pairwise disjoint family $\left(X_{\sigma} ; \sigma<\mu^{\prime}\right)$ of subsets of $\lambda$ such that $\left|X_{\sigma}\right|=\mu_{\sigma}$ for each $\sigma$ less than $\mu^{\prime}$. Suppose that $\sigma<\mu^{\prime}$ and each member of the set $\mathcal{X}_{\sigma}=\left\{X_{\delta} ; \delta<\sigma\right\}$ has been defined. Let $S_{\sigma}=\bigcup\left\{T_{\beta} ; \beta<\Sigma_{\sigma}\right\}$ and let $I_{\sigma}=\left\{\alpha<\lambda ;\left|A_{\alpha} \cap S_{\sigma}\right|=\kappa\right\}$. Then the family $\left(A_{\alpha} \cap S_{\sigma} ; \alpha \in I_{\sigma}\right)$ is an almost disjoint $\left(\left|I_{\sigma}\right|, \kappa\right)$ family of subsets of $S_{\sigma}$ and $\left|I_{\sigma}\right| \leqslant\left|S_{\sigma}\right|^{+}$, where $\left|S_{\sigma}\right|^{+}<\Sigma$ since $\left|S_{\sigma}\right| \leqslant \mu \cdot \Sigma_{\sigma}<\Sigma$ and $\Sigma$ is a limit cardinal. Also, $\left|\cup \mathcal{X}_{\sigma}\right|=\Sigma\left(\mu_{\delta} ; \delta<\sigma\right)<\mu<\Sigma$. Hence $\left|\lambda-\left(I_{\sigma} \cup \cup \mathcal{X}_{\sigma}\right)\right|=\lambda$ and we choose $X_{\sigma}$ from $\left[\lambda-\left(I_{\sigma} \cup \cup \mathcal{X}_{\sigma}\right)\right]^{\mu_{\sigma}}$. Note that if $\alpha \in X_{\sigma}$ then $\left|A_{\alpha} \cap S_{\sigma}\right|<\kappa$. Put $X=\cup\left\{X_{\sigma} ; \sigma<\mu^{\prime}\right\}$. For each $\alpha$ in $X$ let $\sigma(\alpha)$ be the unique $\sigma$ less than $\mu^{\prime}$ such that $\alpha \in X_{\sigma}$ and set $S=\cup\left\{A_{\alpha} \cap\right.$ $\left.S_{\sigma(\alpha)} ; \alpha \in X\right\}$. Since $S$ is the union of $\mu$ sets each of power less than $\kappa$ and $\mu<\kappa^{\prime}$, it follows that $|S|<\kappa$. Hence $\left(A_{\alpha}-S ; \alpha \in X\right)$ is an almost disjoint $(\mu, \kappa)$ family and we choose $T$ to be a $\mu$-sized transversal of this family. This defines $X$ and $T$.

Since $T$ is a transversal of $\left(A_{\alpha}-S ; \alpha \in X\right)$ and $A_{\alpha} \cap T=\left(A_{\alpha}-S\right) \cap T$ for each $\alpha$ in $X$, it follows that $T$ is a transversal of $\mathscr{E}[X]$. To show that $T$ is almost disjoint from each member of $\mathscr{T}$ suppose that $\beta<\Sigma$ and let $\delta(\beta)$ be the least $\delta$ less than $\mu^{\prime}$ such that $\beta<\Sigma_{\delta}$. If $\delta(\beta) \leqslant \sigma<\mu^{\prime}$ then $\left(A_{\alpha} \cap T\right) \cap T_{\beta}=\varnothing$ for 
each $\alpha$ in $X_{0}$. To prove this we argue by contradiction. Suppose that $\delta(\beta) \leqslant \sigma<\mu^{\prime}$, $\alpha \in X_{\sigma}$ and $t \in\left(A_{\alpha} \cap T\right) \cap T_{\beta}$. Then $T_{\beta} \subseteq S_{\sigma}$ since $\beta<\Sigma_{\delta(\beta)} \leqslant \Sigma_{\sigma} ;$ and $t \in A_{\alpha}$ $\cap S_{\sigma}=A_{\alpha} \cap S_{\sigma(\alpha)} \subseteq S$. On the other hand; $t \notin S$ since $t \in T$ and $T \cap S=\varnothing$; a contradiction. Therefore:

$$
\begin{aligned}
T \cap T_{\beta} & =\cup\left\{\left(A_{\alpha} \cap T\right) \cap T_{\beta} ; \alpha \in X\right\} \\
& \subseteq \cup\left\{A_{\alpha} \cap T ; \alpha \in \cup\left\{X_{\sigma} ; \sigma<\delta(\beta)\right\}\right\},
\end{aligned}
$$

and so

$$
\left|T \cap T_{\beta}\right|<\left|\cup\left\{X_{\sigma} ; \sigma<\delta(\beta)\right\}\right|<\mu,
$$

since $\left|A_{\alpha} \cap T\right|=1$ for each $\alpha$ in $X,\left|X_{\sigma}\right|<\mu$ for each $\sigma$ less than $\delta(\beta)$ and $|\delta(\beta)|<\mu^{\prime}$.

This completes the proof of Lemma 2 .

The following two propositions deal with the case when $\Sigma>\kappa$ and $\mu \geqslant \kappa^{\prime}$. Note that $\mathrm{GCH}$ is not required and the family $\mathbb{Q}$ need not be almost disjoint.

Proposition 3. Suppose $\Sigma>\kappa$ and $\mu \geqslant \kappa^{\prime}$. Let $\mathbb{Q}$ be $a(\lambda, \kappa)$ decomposition of $\Sigma$. There exists a pairwise disjoint $(\Sigma, \mu)$ decomposition $\mathcal{T}$ of $\Sigma$ such that each member of $\mathcal{T}$ is a $\mu$-transversal of some $\mu$-sized subfamily of $\mathbb{Q}$.

Proof. Write $\mathscr{Q}=\left(A_{\alpha} ; \alpha<\lambda\right)$. The conditions on the cardinals imply that $\lambda \geqslant \Sigma$. Let $\left\langle\mu_{\sigma} ; \sigma<\mu^{\prime}\right\rangle$ be a $\mu$-sequence and let $\left(L_{\sigma} ; \sigma<\mu^{\prime}\right)$ be a pairwise disjoint $\left(\mu^{\prime}, \Sigma\right)$ decomposition of $\Sigma$.

We inductively define families $\left(X_{\alpha} ; \alpha<\Sigma\right)$ and $\left(T_{\alpha} ; \alpha<\Sigma\right)$ of sets such that $\Sigma$,

(i) $X_{\alpha} \in[\lambda]^{\mu}, T_{\alpha} \in[\Sigma]^{\mu}$ and $T_{\alpha}$ is a $\mu$-transversal of $\mathbb{E}\left[X_{\alpha}\right]$ for each $\alpha$ less than

(ii) $T_{\beta} \cap T_{\alpha}=\varnothing$ if $\beta<\alpha<\Sigma$, and

(iii) $\left|T_{\alpha} \cap L_{\sigma}\right| \leqslant \mu_{\sigma}$ if $\langle\alpha, \sigma\rangle \in \Sigma \times \mu^{\prime}$.

Suppose that $\alpha<\Sigma$ and $X_{\beta}, T_{\beta}$ have been defined for each $\beta$ less than $\alpha$. Let $\mathscr{X}_{\alpha}=\left\{X_{\beta} ; \beta<\alpha\right\}$ and let $\mathscr{T}_{\alpha}=\left\{T_{\beta} ; \beta<\alpha\right\}$. Note that, for each $\sigma$ less than $\mu^{\prime}$,

$$
\left|L_{\sigma} \cap \cup \mathcal{T}_{\alpha}\right|=\left|\cup\left\{T_{\beta} \cap L_{\sigma} ; \beta<\alpha\right\}\right| \leqslant \mu_{\sigma} \cdot|\alpha|<\Sigma,
$$

and $\left|L_{\sigma}-\cup \mathcal{J}_{\alpha}\right|=\Sigma$. For each $\sigma$ less than $\mu^{\prime}$ let

$$
I_{\sigma}=\left\{\alpha<\lambda ; A_{\alpha} \cap\left(L_{\sigma}-\cup \mathcal{T}_{\alpha}\right) \neq \varnothing\right\} .
$$

Since $\Sigma>\kappa$ it follows that $\left|I_{\sigma}\right| \geqslant \Sigma$ for each $\sigma$ less than $\mu^{\prime}$.

To define $X_{\alpha}$ and $T_{\alpha}$ we inductively define two pairwise disjoint families $\left(Y_{\sigma} ; \sigma<\mu^{\prime}\right)$ and $\left(S_{\sigma} ; \sigma<\mu^{\prime}\right)$ such that $\left|Y_{\sigma}\right|=\left|S_{\sigma}\right|=\mu_{\sigma}$ for each $\sigma$ less than $\mu^{\prime}$. Suppose that $\sigma<\mu^{\prime}$ and $Y_{\delta}, S_{\delta}$ have been defined for each $\delta$ less than $\sigma$. Let $\mathscr{Y}_{\sigma}=\left\{Y_{\delta} ; \delta<\sigma\right\}$ and let $\mathcal{S}_{\sigma}=\left\{S_{\delta} ; \delta<\sigma\right\}$. To define $Y_{\sigma}$ and $S_{\sigma}$ we inductively define sequences $\left\langle y^{\sigma}(\gamma) ; \gamma<\mu_{\sigma}\right\rangle,\left\langle s^{\sigma}(\gamma) ; \gamma<\mu_{\sigma}\right\rangle$ of pairwise distinct elements 
of $\lambda, \Sigma$ respectively. Suppose that $\gamma<\mu_{\sigma}$ and $y^{\sigma}(\nu) s^{\sigma}(\nu)$ have been defined for each $\nu$ less than $\gamma$; and let

$$
L_{\sigma}(\gamma)=\left(L_{\sigma}-\cup \mathcal{T}_{\sigma}\right)-\left(\cup \mathcal{S}_{\sigma} \cup \cup \mathfrak{Q}\left[\cup \mathcal{S}_{\sigma}\right] \cup\left\{s^{\sigma}(\nu) ; \nu<\gamma\right\}\right) .
$$

Then $\left|L_{\sigma}(\gamma)\right|=\Sigma$ since

$$
\begin{gathered}
\left|\cup \delta_{\boldsymbol{\sigma}}\right|=\Sigma\left(\mu_{\delta} ; \delta<\sigma\right)<\mu \leqslant \Sigma, \\
\left|\cup \mathfrak{Q}\left[\cup \delta_{\boldsymbol{\sigma}}\right]\right| \leqslant \kappa \cdot\left|\cup \delta_{\boldsymbol{\sigma}}\right|<\Sigma,
\end{gathered}
$$

$\left|L_{\sigma}-\cup \mathcal{G}_{\sigma}\right|=\Sigma$ and $|\gamma|<\mu_{\sigma}<\Sigma$. Since $\Sigma>\kappa$ the set

$$
I_{\sigma}(\gamma)=\left\{\alpha \in I_{\sigma} ; A_{\alpha} \cap L_{\sigma}(\gamma) \neq \varnothing\right\}
$$

has cardinality at least $\Sigma$. Also

$$
\left|\cup \mathscr{Q}_{\sigma}\right|=\Sigma\left(\mu_{\delta} ; \delta<\boldsymbol{\sigma}\right)<\mu \leqslant \Sigma .
$$

Hence

$$
\left|I_{\sigma}(\gamma)-\left(\cup \mathscr{Y}_{o} \cup\left\{y^{\sigma}(\nu) ; \nu<\gamma\right\}\right)\right|=\left|I_{\sigma}(\gamma)\right| \geqslant \Sigma
$$

and we choose $y^{\sigma}(\gamma)$ from this set. (Hence, $y^{\sigma}(\gamma) \notin \cup \mathscr{Y}_{\sigma}$ and $y^{\sigma}(\gamma) \neq y^{\sigma}(\nu)$ for any $\nu$ less than $\gamma$.) Since $y^{\sigma}(\gamma) \in I_{\sigma}(\gamma)$ it follows that $A_{y^{\circ}(\gamma)} \cap L_{\sigma}(\gamma) \neq \varnothing$ and we choose $s^{\sigma}(\gamma)$ from this set. (Hence, $s^{\sigma}(\gamma) \notin \cup \mathcal{S}_{\sigma}$ and $s^{\sigma}(\gamma) \neq s^{\sigma}(\nu)$ for any $\nu$ less than $\gamma$.) This defines $y^{\sigma}(\gamma)$ and $s^{\sigma}(\gamma)$. Set $Y_{\sigma}=\left\{y^{\sigma}(\gamma) ; \gamma<\mu_{\sigma}\right\}$ and set $S_{\sigma}=$ $\left\{s^{\sigma}(\gamma) ; \gamma<\mu_{\sigma}\right\}$. Put $X_{\alpha}=\bigcup\left\{Y_{\sigma} ; \sigma<\mu^{\prime}\right\}$ and put $T_{\alpha}=\bigcup\left\{S_{\sigma} ; \sigma<\mu^{\prime}\right\}$.

The sets $X_{\alpha}, T_{\alpha}$ will do. Since $\left|Y_{\sigma}\right|=\left|S_{\sigma}\right|=\mu_{\sigma}$ for each $\sigma$ less than $\mu^{\prime}$ and the cardinals $\mu_{\sigma}$ sum to $\mu$, it follows that $\left|X_{\alpha}\right|=\left|T_{\alpha}\right|=\mu$. We show that $T_{\alpha}$ is a $\mu$-transversal of $\mathscr{Q}\left[X_{\alpha}\right]$. Now $X_{\alpha}=\left\{y^{\sigma}(\gamma) ; \sigma<\mu^{\prime}\right.$ and $\left.\gamma<\mu_{\sigma}\right\}$ and $s^{\sigma}(\gamma) \in A_{y^{\circ}(\gamma)}$ always. Hence $T_{\alpha} \subseteq \cup \mathbb{Q}\left[X_{\alpha}\right]$ and $T_{\alpha} \cap A_{y} \neq \varnothing$ for each $y$ in $X_{\alpha}$. Next, suppose that $\sigma<\mu^{\prime}$ and $\gamma<\mu_{\sigma}$. If $\sigma<\delta<\mu^{\prime}$ and $\varepsilon<\mu_{\delta}$ then $s^{\delta}(\varepsilon) \notin A_{y^{\circ}(\gamma)}$ since $A_{y^{\sigma}(\gamma)} \subseteq \cup \mathfrak{Q}\left[S_{\delta}\right], s^{\delta}(\varepsilon) \in L_{\delta}(\varepsilon)$ and $L_{\delta}(\varepsilon) \cap \cup \mathcal{Q}\left[S_{\delta}\right]=\varnothing$. Hence

$$
\left|T_{\alpha} \cap A_{y^{\sigma}(\gamma)}\right| \leqslant\left|\cup\left\{S_{\delta} ; \delta \leqslant \sigma\right\}\right|=\sum\left(\mu_{\delta} ; \delta \leqslant \sigma\right)<\mu,
$$

and $T_{\alpha}$ is a $\mu$-transversal of $\mathscr{Q}\left[X_{\alpha}\right]$ as claimed. If $\beta<\alpha$ then $T_{\beta} \cap T_{\alpha}=\varnothing$ since $T_{\alpha} \subseteq \Sigma-\cup \mathcal{F}_{\alpha}$. Finally, if $\sigma<\mu^{\prime}$ then $T_{\alpha} \cap L_{\sigma}=S_{\sigma}$ and $\left|T_{\alpha} \cap L_{\sigma}\right| \leqslant \mu_{\sigma}$ as required. This completes the construction of $X_{\alpha}$ and $T_{\alpha}$.

The family $\mathscr{T}=\left(T_{\alpha} ; \alpha<\Sigma\right)$ is a pairwise disjoint family of $\mu$-sized subsets of $\Sigma$ and each member of $\mathcal{T}$ is a $\mu$-transversal of some $\mu$-sized subfamily of $\mathbb{Q}$.

The next proposition is a modification of Proposition 3 and gives a related result in the case when $\mu^{\prime}=\Sigma^{\prime}$.

Proposition 4. Suppose $\Sigma>\kappa, \mu \geqslant \kappa^{\prime}$ and $\mu^{\prime}=\Sigma^{\prime}$. Let $Q$ be $a(\lambda, \kappa)$ decomposition of $\Sigma$. There exists an almost disjoint $\left(\Sigma^{+}, \mu\right)$ decomposition $\mathcal{T}$ of $\Sigma$ such that each member of $\mathcal{T}$ is a $\mu$-transversal of some $\mu$-sized subfamily of $\mathbb{Q}$. 
Proof. The proof involves only minor modifications to the proof of Proposition 3 to deal with the inductive step when $\left|\mathscr{T}_{\alpha}\right|=\Sigma$. We refer to the proof of Proposition 3 for details. Write $Q=\left(A_{\alpha} ; \alpha<\lambda\right)$. Let $\left\langle\mu_{\sigma} ; \sigma<\mu^{\prime}\right\rangle$ be a $\mu$ sequence and let $\left\langle\Sigma_{\sigma} ; \sigma<\mu^{\prime}\right\rangle$ be a $\Sigma$-sequence. Suppose $\mathscr{N}=\left(M_{\sigma} ; \sigma<\mu^{\prime}\right)$ is a pairwise disjoint decomposition of $\Sigma$ such that $\left|M_{\sigma}\right|=\Sigma_{\sigma}$ for each $\sigma$ less than $\mu^{\prime}$. Let $\left(L_{\sigma} ; \sigma<\mu^{\prime}\right)$ be a pairwise disjoint $\left(\mu^{\prime}, \Sigma\right)$ decomposition of $\Sigma$. As in Proposition 3 , we inductively construct families $\left(X_{\alpha} ; \alpha<\Sigma^{+}\right)$and $\left(T_{\alpha} ; \alpha<\Sigma^{+}\right)$such that

(i) $X_{\alpha} \in[\lambda]^{\mu}, T_{\alpha} \in[\Sigma]^{\mu}$ and $T_{\alpha}$ is a $\mu$-transversal of $\mathbb{Q}\left[X_{\alpha}\right]$ for each $\alpha$ less than $\Sigma^{+}$

(ii) $\left|T_{\beta} \cap T_{\alpha}\right|<\mu$ if $\beta<\alpha<\Sigma^{+}$,

(iii) $\left|T_{\alpha} \cap L_{\sigma}\right| \leqslant \mu_{\sigma}$ if $\langle\alpha, \sigma\rangle \in \Sigma^{+} \times \mu^{\prime}$.

The families $\left(X_{\alpha} ; \alpha<\Sigma\right)$ and $\left(T_{\alpha} ; \alpha<\Sigma\right)$ were constructed in Proposition 3. Next, suppose that $\Sigma \leqslant \alpha<\Sigma^{+}$and $X_{\beta}, T_{\beta}$ have been defined for each $\beta$ less than $\alpha$. The families $\mathscr{X}_{\alpha}, \mathscr{T}_{\alpha}$ are as before and we re-index $\mathscr{T}_{\alpha}$ by the ordinals $\varepsilon$ less than $\Sigma$ : write $\widetilde{T}_{\alpha}=\left(\underline{T}_{\varepsilon} ; \varepsilon<\Sigma\right)$. The construction of $X_{\alpha}$ and $T_{\alpha}$ is similar to that in Proposition 3 except that here we define

$$
I_{\alpha}=\left\{\alpha<\lambda ; A_{\alpha} \cap\left(L_{\alpha}-\cup\left\{\underline{T}_{\beta} ; \beta \in \cup \Re[\sigma]\right\}\right) \neq \varnothing\right\} .
$$

The sets $\mathcal{S}_{\sigma}$ and $\mathcal{Y}_{\sigma}$ are as before. The construction of $y^{\sigma}(\gamma)$ and $s^{\sigma}(\gamma)$ is similar except that here we define

$$
\begin{aligned}
L_{\sigma}(\gamma)= & \left(L_{\sigma}-\cup\left\{\underline{T}_{\beta} ; \beta \in \cup \mathscr{N}[\sigma]\right\}\right) \\
& -\left(\cup \mathcal{S}_{\sigma} \cup \cup \mathfrak{Q}\left[\cup \mathcal{S}_{\sigma}\right] \cup\left\{s^{\sigma}(\nu) ; \nu<\gamma\right\}\right) .
\end{aligned}
$$

The sets $X_{\alpha}$ and $T_{\alpha}$ have all the required properties. We present only the proof that $\left|T_{\beta} \cap T_{\alpha}\right|<\mu$ for each $\beta$ less than $\alpha$. Suppose $\varepsilon<\Sigma$ and let $\sigma(\varepsilon)$ be the unique $\sigma$ less than $\mu^{\prime}$ such that $\varepsilon \in M_{\sigma}$. If $\sigma(\varepsilon)<\sigma<\mu^{\prime}$ then $T_{\varepsilon} \subseteq \cup\left\{\underline{T}_{\beta} ; \beta \in\right.$ $\cup \mathscr{N}[\sigma]\}$ and $\underline{T}_{\varepsilon} \cap L_{\sigma}(\gamma)=\varnothing$ for all $\gamma$ less than $\mu_{\sigma}$. Hence $\underline{T}_{\varepsilon} \cap S_{\sigma}=\varnothing$ for each $\sigma$ with $\sigma(\varepsilon)<\sigma<\mu^{\prime}$. Therefore, $\underline{T}_{\varepsilon} \cap T_{\alpha} \subset \cup\left\{S_{\sigma} ; \sigma \leqslant \sigma(\varepsilon)\right\}$ and $\left|\underline{T}_{\varepsilon} \cap T_{\alpha}\right|$ $<\mu$ as required.

The family $\mathcal{T}=\left(T_{\alpha} ; \alpha<\Sigma^{+}\right)$is an almost disjoint $\left(\Sigma^{+}, \mu\right)$ decomposition of $\Sigma$ and each member of $\widetilde{T}$ is a $\mu$-transversal of some $\mu$-sized subfamily of $\mathcal{Q}$.

We are now in a position to prove that $R S_{\theta}(\mu, \mathbb{Q})=S_{\theta}(\mu, \Sigma)$.

Proof of Theorem. Write $\mathscr{Q}=\left(A_{\alpha} ; \alpha<\lambda\right)$. Clearly, $R S_{\theta}(\mu, \mathscr{Q}) \leqslant S_{\theta}(\mu, \Sigma)$. Hence

(a) if $\theta<\mu$ or if $\mu^{\prime} \neq \Sigma^{\prime}$, then $R S_{\theta}(\mu, \mathbb{Q}) \leqslant \Sigma$.

(b) If $\mu^{\prime}=\Sigma^{\prime}$ then $R S(\mu, \mathbb{Q}) \leqslant \Sigma^{+}$. 
To show that these upper bounds are the values of $R S_{\theta}(\mu, \mathbb{Q})$ we construct, in each case, a 'suitably large' family $\mathcal{T}$ of $\mu$-partial representing sets of $\mathbb{Q}$ such that $\delta(\widetilde{T}) \leqslant \theta$.

Case 1. $\kappa=\Sigma$ and $\mu<\kappa$. It is clear that $\mathscr{Q}[\mu]$ possesses a pairwise disjoint $(\kappa, \mu)$ family of representing sets. This suffices if either $\theta<\mu$ or $\mu^{\prime} \neq \kappa^{\prime}$. Next, suppose $\theta=\mu$ and $\mu^{\prime}=\kappa^{\prime}$. Then $\kappa$ is singular and we choose $\left\langle\kappa_{\sigma} ; \sigma<\mu^{\prime}\right\rangle$ to be a strictly increasing $\kappa$-sequence. Let $\left\langle\mu_{\sigma} ; \sigma<\mu^{\prime}\right\rangle$ be a $\mu$-sequence. Inductively define an almost disjoint family $\left(T_{\alpha} ; \alpha<\kappa^{+}\right)$of $\mu$-sized representing sets of $\mathscr{Q}[\mu]$ as follows. Suppose that $\alpha<\kappa^{+}$and the members of $\sigma_{\alpha}=\left(T_{\beta} ; \beta<\alpha\right)$ have been defined. Write $\sigma_{\alpha}=\left(T_{\varepsilon} ; \varepsilon<\kappa\right)$ (Repetitions occur if $\left.\alpha<\kappa\right)$. To define $T_{\alpha}$ inductively define a pairwise disjoint family of subsets of $\kappa$ with $\left|S_{\sigma}\right|=\mu_{\sigma}$ for all $\sigma$ less than $\mu^{\prime}$ as follows. Given $\sigma$ less than $\mu^{\prime}$ choose $S_{\sigma}$ to be a $\mu_{\sigma}$-sized representing set of the almost disjoint $\left(\mu_{\sigma}, \kappa\right)$ family

$$
\left(A_{\nu}-\left(\cup\left\{\underline{T}_{\varepsilon} ; \varepsilon<\kappa_{\sigma}\right\} \cup \cup\left\{S_{\delta} ; \delta<\sigma\right\}\right) ; \nu<\mu_{\sigma}\right),
$$

and set $T_{\alpha}=\bigcup\left\{S_{\sigma} ; \sigma<\mu^{\prime}\right\}$. The set $T_{\alpha}$ will do. Then $\mathscr{T}=\left(T_{\alpha} ; \alpha<\kappa^{+}\right)$is an almost disjoint $\left(\kappa^{+}, \mu\right)$ family of $\mu$-partial representing sets of $\mathscr{Q}$ and the result follows in this case.

Case 2. $\kappa=\Sigma$ and $\mu=\kappa$. The proof is immediate from Balanda [1]. Let $\mathscr{T}$ be a family of $\kappa$-sized representing sets of $\mathbb{Q}[\kappa]$ with $\delta(\mathcal{T}) \leqslant \theta$ and $|\mathcal{T}|=S_{\theta}(\kappa, \kappa)$. The family $\mathscr{T}$ consists of $\mu$-partial representing sets of $\mathcal{Q}$ and the result follows in this case.

Case 3. $\Sigma>\kappa$. In this case we use the lemmas and propositions above. First suppose that $\mu<\kappa^{\prime}$. A simple application of Zorn's Lemma shows there is a family $\mathscr{T}$ of $\mu$-partial representing sets of $\mathscr{Q}$ that is maximal with respect to $\delta(\mathscr{T}) \leqslant \theta$. Lemmas 1 and 2 guarantee that $|\mathscr{T}| \geqslant \Sigma$ if $\theta<\mu$ or if $\mu^{\prime} \neq \Sigma^{\prime}$, and $|\mathscr{T}| \geqslant \Sigma^{+}$if $\theta=\mu$ and $\mu^{\prime}=\Sigma^{\prime}$. Next, suppose that $\mu \geqslant \kappa^{\prime}$. Propositions 3 and 4 show that there exists a $\left(S_{\theta}(\mu, \Sigma), \mu\right)$ family $\mathscr{T}$ with $\delta(\mathscr{T}) \leqslant \theta$ such that each member of $\mathscr{T}$ is a $\mu$-transversal of a $\mu$-sized subfamily of $Q$.

This completes the proof of the Theorem.

\section{References}

[1] K. P. Balanda, 'Maximally almost disjoint families of representing sets', Math. Proc. Cambridge Philos. Soc. 93 (1983), 1-7.

[2] K. P. Balanda, 'Almost disjoint families of representing sets', Z. Math. Logik Grundlag. Math., to appear.

[3] J. E. Baumgartner, 'Almost-disjoint sets, the dense set problem and partition calculus', Ann. Math. Logic 9 (1976), 401-439. 
[4] P. Erdös and R. Rado, 'Intersection theorems for systems of sets $I$ ', J. London Math. Soc. 44 (1969), 467-479.

[5] N. H. Williams, Combinatorial set theory (North-Holland, Amsterdam, 1977).

Department of Mathematics

University of Queensland

St. Lucia, Qld 4067

Australia 